\title{
Tuberculosis infection in foreign-born children: a screening survey based on skin and blood testing
}

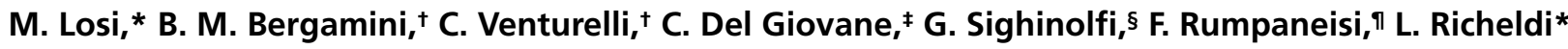 \\ * Section of Respiratory Diseases, Department of Oncology, Haematology and Respiratory Diseases, ${ }^{\dagger}$ Department of \\ Paediatrics, and ₹Biostatistics Unit, Department of Oncology, Haematology and Respiratory Diseases, University of \\ Modena and Reggio Emilia, Modena, \$Community Paediatrics Unit, Primary Care Trust, Local Health Service of \\ Modena, Modena, ๆ Microbiology and Virology Laboratory, University-Hospital Policlinico of Modena, Modena, Italy
}

This study, carried out in a low tuberculosis (TB) prevalence country with high immigration rates from high TB prevalence countries, deals with the interferon-gamma release assay, QuantiFERON ${ }^{\circledR}$-TB Gold In-Tube, for the diagnosis of latent TB infection (LTBI) in foreign-born children. The results of our study highlight the poten- tial advantages and concerns of using a blood test for diagnosing LTBI in a 'two-step' strategy in foreign-born children.

KEY WORDS: latent tuberculosis infection; foreignborn children; active tuberculosis; interferon-gamma assays
OVER THE LAST 30 YEARS, industrialised countries have witnessed a progressive increase in tuberculosis (TB) incidence, partly related to the growing number of immigrants from high TB incidence areas. Accurate diagnosis of latent TB infection (LTBI) cases is a desirable goal in achieving effective TB control in low-incidence countries, particularly in young children, who are more prone to severe disease as a consequence of recent infection and in whom preventive chemotherapy is effective and relatively safe..$^{1,2}$

Until recently, LTBI detection relied only on the tuberculin skin test (TST), known to be associated with false-positive results in children infected with nontuberculous mycobacteria (NTM) or bacille CalmetteGuerin (BCG) vaccinated, as is the case with the majority of the children born in high TB incidence countries. $^{3,4}$ Furthermore, TST sensitivity may be impaired in young children, and in several other conditions such as immunosuppression. 5,6 The positive cut-off of the TST indura-tion therefore depends on the likelihood of infection and on the individual risk of developing active TB.6,7 Interferon-gamma release assays (IGRAs) represent an attractive tool for TB screening due to their high diagnostic accuracy, although their performance in paediatric subjects needs corroborating evidence.

Policies for TB screening in immigrant children vary widely across Europe. ${ }^{8}$ For immigrants from TBendemic countries, the current Italian national guidelines set the cut-off for a positive TST at $10 \mathrm{~mm}$, regardless of age and BCG vaccination status.
Given the increasing numbers of active TB cases among children and adolescents in recent years in our region, in late 2005 the community paediatrics unit of the local health service in Modena implemented testing with the QuantiFERON ${ }^{\circledR}$-TB Gold In-Tube assay (QFT; Cellestis Ltd, Carnegie, VIC, Australia) of all children with both positive $(\geqslant 10 \mathrm{~mm})$ and borderline (5-9 mm) TST ( 5 units of purified protein derivative, Biocine Test PPD, Chiron, Siena, Italy). ${ }^{9}$ Samples for IGRA testing were processed according to the manufacturer's instructions. In the present study, we report data on a group of immigrant children evaluated between January 2006 and December 2008.

\section{RESULTS}

We performed 621 TSTs in children and adolescent immigrants, mostly from Africa (40.3\%) and Eastern Europe $(26.9 \%)$ : 104 (16.7\%) were TST-positive, $128(20.6 \%)$ were TST-borderline and 389 (62.6\%) TST-negative. Children in the first two groups were tested with QFT at $31 \pm 29$ days (mean \pm standard deviation) after TST. Three children had an invalid QFT result and were excluded from further analyses. QFT was positive in $80(34.5 \%)$ children: $30 \mathrm{had}$ borderline TST $(30 / 125,24 \%)$ and 50 were TST-positive (50/104, 48.1\%; Figure 1). By logistic regression analysis, a positive correlation was found between a positive QFT and the size of TST induration (odds ratio [OR] 1.23, 95\% confidence interval [CI] 1.12$1.36, P<0.001)$.

Correspondence to: Monica Losi, Section of Respiratory Diseases, Department of Oncology, Haematology and Respiratory Diseases, University of Modena and Reggio Emilia, Via del Pozzo 71 Modena 41124, Italy. Tel: (+39) 059 422 $3469 / 2198$. Fax: (+39) 059422 4231. e-mail: monica.losi@unimore.it 


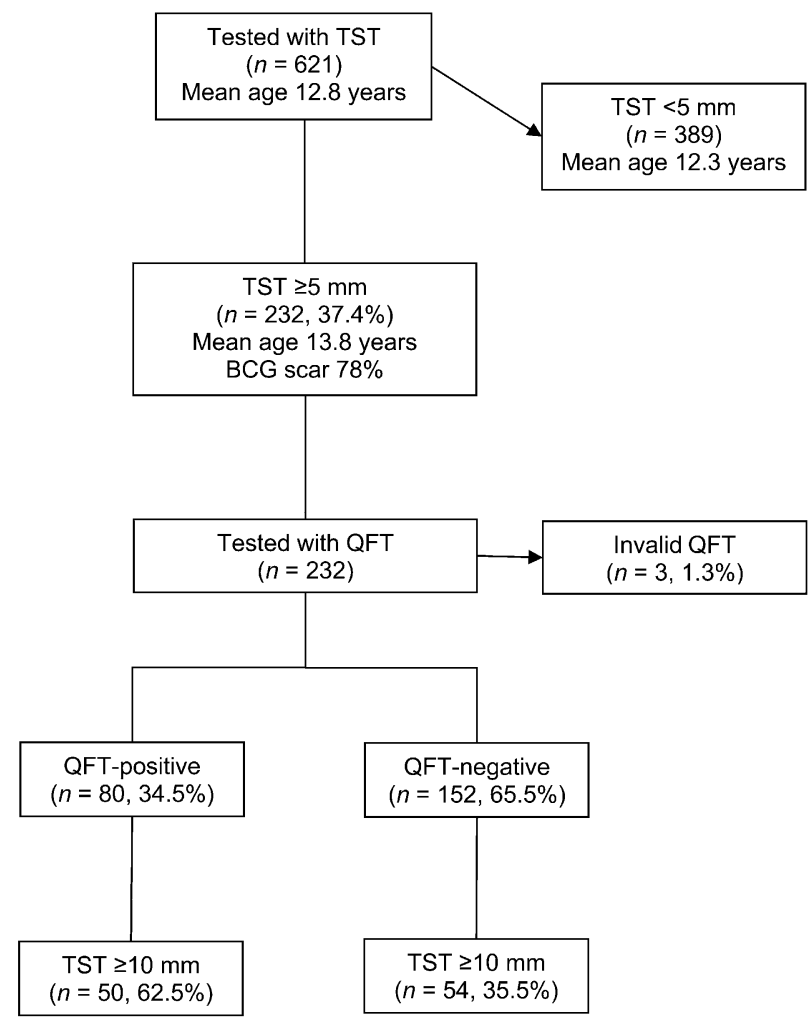

Figure 1 Flow chart of the study. TST = tuberculin skin test: BCG $=$ bacille Calmette-Guérin; QFT $=$ QuantiFERON ${ }^{\circledR}-\mathrm{TB}$ Gold In-Tube.

The distribution of TST and QFT results according to age is shown in Figure 2. TST-positive, QFTnegative results diminish with increasing age $(\mathrm{OR}$ $0.88,95 \%$ CI $0.82-0.95, P=0.001$ ), while TSTborderline, QFT-positive results are not related to age (OR 1.04, 95\% CI 0.94-1.15, P = 0.458). All QFTnegative, TST-positive tests had interferon-gamma $($ IFN- $\gamma$ ) levels $<0.20$ international units $(\mathrm{IU}) / \mathrm{ml}$, while the majority $(n=26)$ of the 30 QFT-positive children with borderline TST had IFN- $\gamma$ levels $>0.50 \mathrm{IU} / \mathrm{ml}$.

All children were healthy on clinical examination and none complained of respiratory symptoms. Nevertheless, four TST-positive, QFT-positive children had radiological findings consistent with active $\mathrm{TB}$, confirmed by microbiological culture (acid-fast bacilli smear-negative). These TB cases were aged $>10$ years and BCG-vaccinated: TST size and IFN- $\gamma$ levels were respectively $12 \mathrm{~mm} / 17.0 \mathrm{IU}, 13 \mathrm{~mm} / 15.9 \mathrm{IU}, 11 \mathrm{~mm} /$ $16.2 \mathrm{IU}$ and $10 \mathrm{~mm} / 9.4 \mathrm{IU}$. Isoniazid (INH) was offered to all QFT-positive children $(n=76)$, with the exception of two children with coexisting chronic liver disease. Based on clinical judgment, children aged $<8$ years with TST $>15 \mathrm{~mm}(n=3)$ were also treated with INH, as were those subjects for whom reliable follow-up could not be guaranteed $(n=4)$. For TST-positive, QFT-negative children, paediatricians were advised to provide strict follow-up; at the time of writing, no additional cases of active TB have been notified in this group.

\section{DISCUSSION}

Using blood tests to diagnose LTBI in immigrant children permits the diagnosis of infection in a sizeable fraction of children with TST results between 5 and $9 \mathrm{~mm}$ : we report here that about one third of these children were QFT-positive, usually with high IFN- $\gamma$ levels. Although longitudinal data in children are not yet available, it is currently assumed that individuals with TST induration between 5 and $9 \mathrm{~mm}$ and a positive QFT are likely to be infected with Mycobacterium tuberculosis. ${ }^{10}$ Based on this assumption, we offered treatment to all of these children. Assuming that children with a positive QFT are truly infected and that those with a negative TST are non-infected, LTBI

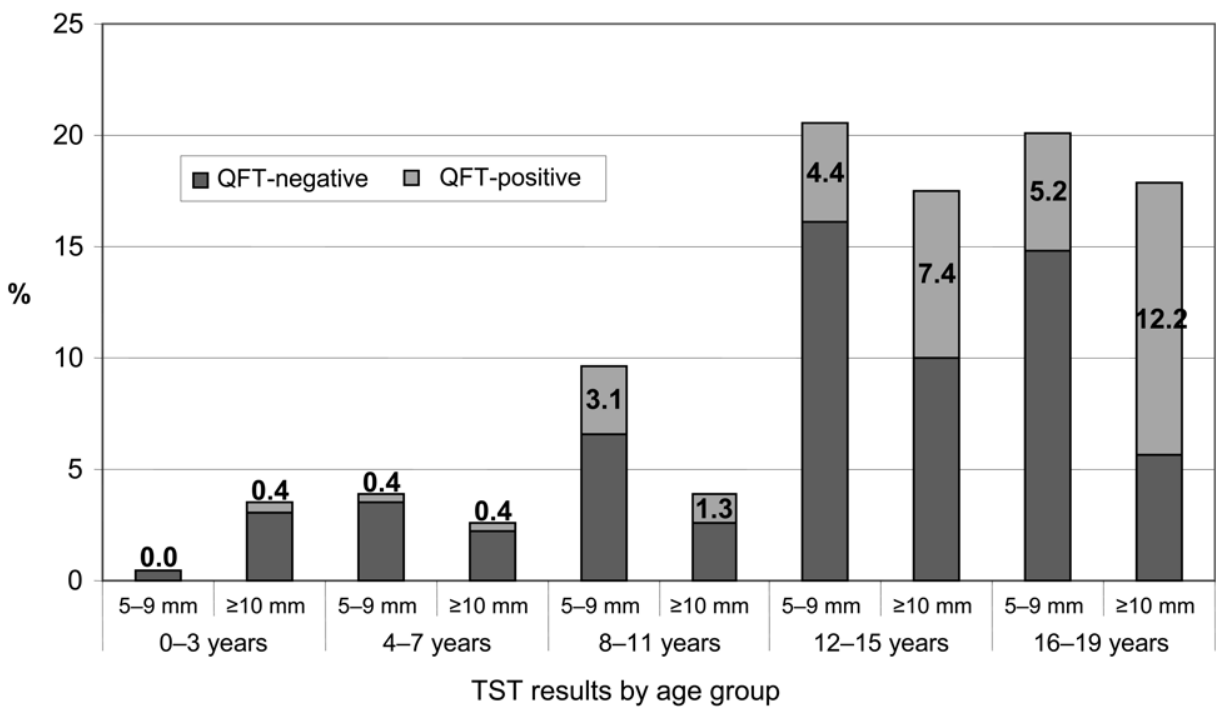

Figure 2 Distribution of QFT results by TST result and age group. TST = tuberculin skin test: QFT = QuantiFERON ${ }^{\circledR}$-TB Gold In-Tube. 
prevalence in this group could be estimated to be around $13 \%$. A limitation of this study is that TSTnegative children did not undergo QFT testing, and we cannot exclude that some of them might also be infected.

As previously reported, only half of the children with a positive TST were QFT-positive. Both BCG vaccination and exposure to NTM might help in interpreting some of these TST results as falsely positive; recent longitudinal data in household contacts would support this concept. ${ }^{11}$ Active pulmonary TB was diagnosed in four BCG-vaccinated, asymptomatic adolescents: all were positive on both QFT (at high IFN- $\gamma$ levels) and TST $(>10 \mathrm{~mm})$. These findings confirm recent data obtained in the context of contact screening showing that quantitative results of both TST and IGRA may assist in the identification of subjects with active disease. ${ }^{12,13}$ A combination of a TST $>10 \mathrm{~mm}$ and high QFT levels might also help to identify children with active disease in the context of screening programmes.

BCG vaccination is usually given in the first days of life, and its effect on TST is presumed to be strongest in the first $10-15$ years. ${ }^{4}$ On the other hand, younger children are more prone to developing severe disease. As the sensitivity of IGRA in young children has not yet been proven, the decision to disregard a positive TST because of a negative QFT is debatable, and caution should be used in this regard. It is therefore crucial to emphasise the importance of parental education regarding symptoms of TB. Consistent clinical follow-up to monitor for disease progression in young children should be in place, particularly considering the poor (TST) or still unproven (IGRA) sensitivity of diagnostic tests in this population group. Last, our data indicate that clinicians must always consider age and risk of disease progression when deciding whether to disregard a positive TST in young immigrant children.

\section{References}

1 World Health Organization. Guidance for national tuberculosis programmes on the management of tuberculosis in children. WHO/HTM/TB/2006.371. WHO/FCH/CAH/2006.7. Geneva, Switzerland: WHO, 2006.

2 Tuberculosis Collaborative Group Infection in Children and Adolescents. Targeted tuberculin skin testing and treatment of latent tuberculosis. Pediatrics 2004; 114: 1175-1201.

3 Farhat M, Greenaway C, Pai M, Menzies F. False-positive tuberculin skin test: what is the absolute effect of BCG and nonmycobacteria? Int J Tuberc Lung Dis 2006; 10: 1192-1204.

4 Wang L, Turner M O, Elwood R K, Schulzer M, FitzGerald J M. A meta-analysis of the effect of bacille Calmette Guerin vaccination on tuberculin skin test measurement. Thorax 2002; 57: 804-809.

5 Lewinsohn D, Gennaro M, Scholvinck D. Tuberculosis immunology in children: diagnostic and therapeutic challenges and opportunities. Int J Tuberc Lung Dis 2004; 8: 658-674.

6 Huebner R E, Schein M F, Bass J B. The tuberculin skin test. Clin Infect Dis 1993; 17: 968-975.

7 American Thoracic Society. Diagnostic standards and classification of tuberculosis in adults and children. Am J Respir Crit Care Med 2000; 161: 1376-1395.

8 Alvarez G G, Clark M, Altper E, et al. Paediatric tuberculosis immigration screening in high-immigration, low-incidence countries. Int J Tuberc Lung Dis 2010; 14: 1530-1537.

9 Comstock G W, Edwards L B, Philip R N, Winn W A. A comparison in the United States of America of two tuberculins, PPD-S and RT 23. Bull World Health Organ 1964; 31: 161-170.

10 Pai M, Dendukuri N, Wang L, Jashi R, Kalantri S, Rieder H L. Improving the estimation of tuberculosis infection prevalence using T-cell-based assay and mixture models. Int J Tuberc Lung Dis 2008; 12: 895-902.

11 Diel R, Goletti D, Ferrara G, et al. Interferon- $\gamma$ release assays for the diagnosis of latent Mycobacterium tuberculosis infection: a systematic review and meta-analysis. Eur Respir J 2011; 37: 88-99.

12 Diel R, Loddenkemper R, Meywald-Walter K, Nienhaus A. Predictive value of a whole blood IFN-gamma assay for the development of active tuberculosis after recent infection with Mycobacterium tuberculosis. Am J Respir Crit Care Med 2008; 177: 1164-1170.

13 Diel R, Loddenkemper R, Meywald-Walter K, Nienhaus A. Negative and positive predictive value of a whole-blood interferon\{gamma\} release assay for developing active tuberculosis: an update. Am J Respir Crit Care Med 2011; 183: 88-95. 
Notre étude, menée dans un pays à faible prévalence de la tuberculose (TB) avec des taux d'immigration élevés provenant de pays à haute prévalence de la TB, traite du test de libération de l'interféron-gamma, QuantiFERON ${ }^{{ }_{-}}$ TB Gold In-Tube pour le diagnostic de l'infection tuberculeuse latente (LTBI) chez les enfants nés à l'étranger.
Les résultats de notre étude illustrent les avantages potentiels et les préoccupations concernant l'utilisation d'un test sanguin pour le diagnostic d'une LTBI dans une stratégie en deux étapes concernant les enfants nés à l'étranger.

RES U M E N

El presente estudio se llevó a cabo en un país de baja prevalencia de tuberculosis con un alto nivel de inmigración procedente de países con tasas altas de prevalencia de esta enfermedad; se analizó el rendimiento de la prueba de liberación de interferón gama, QuantiFERON $^{\circledR}{ }_{-}$TB Gold En Tubo, en el diagnóstico de la in- fección tuberculosa latente (LTBI) en los niños nacidos en el extranjero. Los resultados destacan las posibles ventajas y las dificultades del uso de una prueba sanguínea para establecer el diagnóstico de LTBI en los niños nacidos en el extranjero, mediante una estrategia en dos etapas. 